\title{
Spectral Prediction of Juxtaposed Halftones Relying on the Two-by-Two Dot Centering Model
}

\author{
Vahid Babaei and Roger D. Hersch ${ }^{\wedge}$ \\ Ecole Polytechnique Fédérale de Lausanne (EPFL), Station 14, 1015 Lausanne, Switzerland \\ E-mail:vahid.babaei@epfl.ch
}

\begin{abstract}
In a color reproduction workflow, spectral prediction models are useful for establishing the correspondence between colorant surface coverages and resulting printed halftone color. Spectral prediction models enable calculation of the color gamut and establishment of the color separation tables. Discrete line juxtaposed halftoning, a recently proposed algorithm, is characterized by the fact that colorants formed by inks and ink superpositions are placed side by side. Juxtaposed halftoning is necessary when printing with special inks such as opaque or metallic inks. In order to predict the color of classical halftones, the Yule-Nielsen modified spectral Neugebauer model is generally used. However, this model may not predict the color of juxtaposed halftones, since the effective surface coverages of colorants and of possible colorant overlaps are unknown. In contrast, the two-by-two dot centering spectral prediction model developed by S. G. Wang enables the reflectance of slightly overlapping colorants to be captured and is therefore appropriate for predicting the color of juxtaposed halftones. Since this model requires a large calibration set, the authors use an estimation technique which predicts more than $90 \%$ of the two-by-two calibration pattern reflectances by measuring less than $10 \%$ of them. For juxtaposed halftoning, the two-by-two dot centering model offers high prediction accuracies and outperforms the different variants of the Yule-Nielsen spectral Neugebauer model for comparable setups. (c) 2013 Society for Imaging Science and Technology.
\end{abstract}

[DOI: 10.2352/J.ImagingSci.Technol.2013.57.4.040501]

\section{INTRODUCTION}

Printer characterization is critical for high-quality color reproduction. Characterizing a printer aims at establishing the relationship between input control values such as nominal amounts of inks and the resulting printed color. For given input control values, forward characterization determines the printed color or a related attribute such as the reflectance spectrum. There are several approaches for printer characterization. The first one is a black-box approach relying on color measurements of a relatively large number of printed color samples and on interpolation to create relationships between colors and amounts of inks. The second approach relies on a spectral prediction model accounting for the interaction of light, paper and ink halftones. The parameters of the spectral prediction model are usually derived by measuring a relatively small number of color samples forming the calibration set. ${ }^{1}$

\section{$\triangle$ IS\&T Member.}

Received Jul. 30, 2012; accepted for publication Aug. 14, 2013; published online Jul. 1, 2013. Associate Editor: George Chiu.

1062-3701/2013/57(4)/040501/9/\$20.00
Juxtaposed halftoning is necessary when printing with special inks such as opaque or metallic inks. In a juxtaposed halftone, colorants formed by an ink or by ink superpositions are placed side by side. However, existing color prediction models ${ }^{2}$ assume that the different ink layers are superposed independently. This is a true assumption for classical halftoning methods such as rotated clustered dot halftoning and blue noise dithering. In such halftones, ink dots have overlapping parts which form new colorants, also called Neugebauer primaries, whose surface coverages depend on the surface coverages of the inks. ${ }^{3}$

For juxtaposed halftoning there is no established model specifying how to compute the physical dot gain of the individual colorants and to what extent they possibly overlap. To the best of our knowledge, color prediction of juxtaposed halftones has never been investigated. The two-by-two dot centering spectral prediction model developed by S. G. Wang enables the reflectance of slightly overlapping colorants to be captured and is therefore appropriate for predicting the color of juxtaposed halftones. In this article, we adapt the two-by-two dot centering model to discrete line juxtaposed halftones. A comparison between the two-by-two model and models that are suitable only for classical halftones is also provided. Moreover, we explain the relationship between the $n$-values used by the two-by-two model and by the Yule-Nielsen spectral Neugebauer model (YNSN). Furthermore, we extend the spectral predictions to a larger number of custom inks, i.e., cyan, magenta, yellow, custom blue, custom red, custom green and custom achromatic black.

\section{DISCRETE LINE JUXTAPOSED HALFTONING}

In the past, several juxtaposed halftoning algorithms have been proposed. ${ }^{5-7}$ They were either not able to create multichromatic clustered dots $^{5}$ or were limited to a three-ink system. ${ }^{6}$ The present authors introduced a new juxtaposed halftoning algorithm which creates side by side laid out colorant halftone line screens without limiting the number of colorants. ${ }^{8}$ The proposed juxtaposed halftoning method relies on discrete line geometry that provides subpixel precision for creating discrete line screens.

Based on the definition of a discrete line introduced by Reveillès, ${ }^{9}$ discrete line juxtaposed halftoning enables the creation of discrete lines of subpixel thickness having any desired rational thickness and orientation. The screen elements 


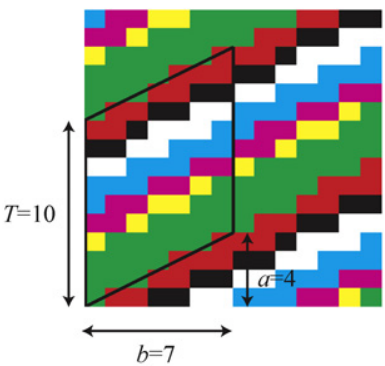

(a)

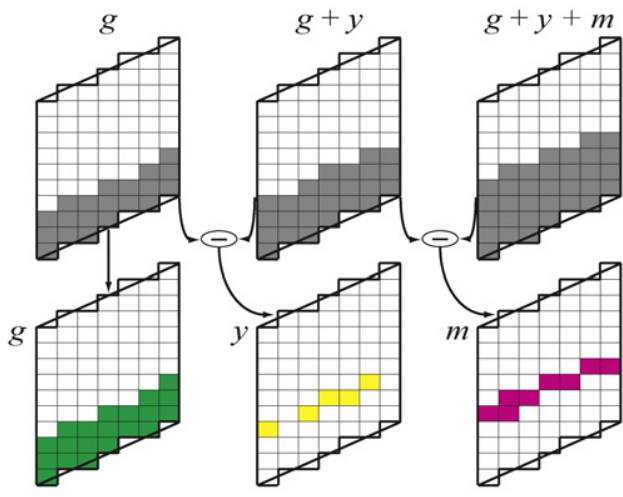

(b)

Figure 1. (a) A juxtaposed halftone line screen of orientation $a / b=4 / 7$ and vertical thickness $T=10$ with the seven colorants cyan, magenta, yellow, green, red, black and white. The corresponding parallelogram screen element boundary is shown as a solid line. (b) Synthesis of three colorants within the screen element by accessing the black/white screen element library: green is directly taken from the library, yellow is synthesized from the sum of green and yellow surface coverages minus the surface coverage of green, and magenta is synthesized from the sum of green, yellow and magenta surface coverages minus the surface coverage of green and yellow.

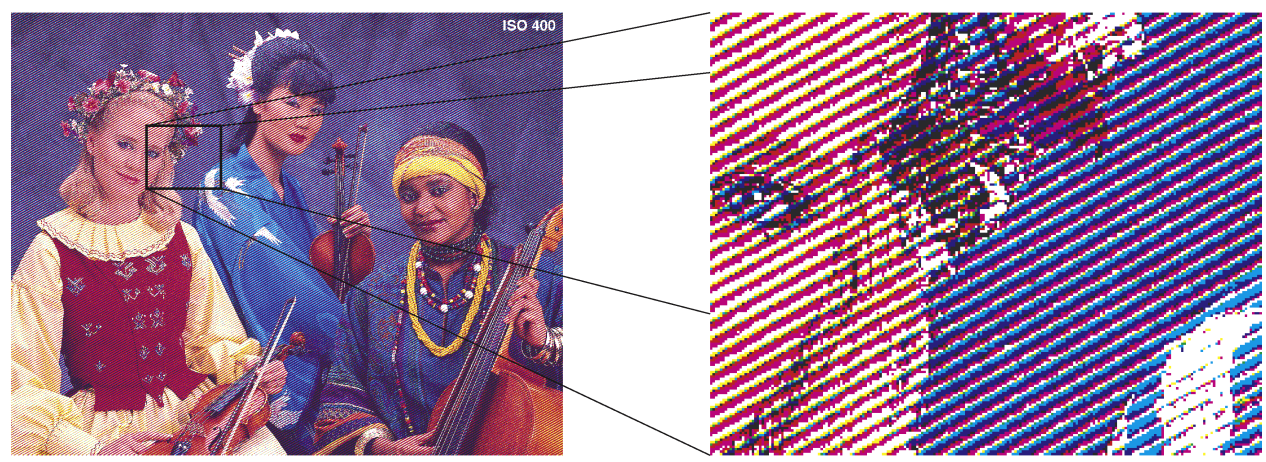

Figure 2. An example of discrete line juxtaposed halftoning with parameters $a / b=4 / 7$ and $T=15$ (for details, see the electronic version).

are formed by discrete line segments whose thicknesses are set according to the desired colorant surface coverages (see appendix A). The maximum thickness $T$ of the line screen element determines the screen period and the discrete line slope $a / b$ defines the orientation of the screen element. Hence, for a black and white halftone, the screen element is a discrete parallelogram whose surface is segmented into black and white parts according to the desired ratio of black and white surface coverages. Black discrete lines at all possible thicknesses (from 0 to $T$ ) form the binary screen element library.

For a color halftone, the parallelogram screen element is composed of successive discrete line segments, each having its predefined colorant color. Since a fully populated color screen element library would require a huge space, we construct only a library of black and white halftones. This library is accessed at halftoning time when a new discrete line segment of a given colorant needs to be generated. Figure 1(a) shows an example of a juxtaposed halftone screen incorporating seven discrete line colorants placed side by side. The parallelogram screen element tile is also shown. Fig. 1(b) shows how a multi-color screen element can be generated from the black/white screen elements of the screen element library. Figure 2 shows an example of a color halftone image created by discrete line halftoning.

\section{SPECTRAL PREDICTION MODELS}

In this section, we review the spectral prediction models that are used in our experiments. We present the two-by-two dot centering model which is a halftone independent spectral prediction model and its predictive variant which relies on a smaller set of calibration samples. In order to evaluate the proposed models, we compare their prediction accuracy with that obtained by the well-known Yule-Nielsen modified spectral Neugebauer model (YNSN) and its variants, using "Demichel compatible" surface coverages for the same printer, paper and inks.

\section{The Two-by-Two Dot Centering Model}

About 15 years ago, Wang ${ }^{10}$ proposed the two-by-two dot centering spectral prediction model. It assumes that each halftone can be represented by an assembly of a limited number of representative two-by-two pixel patterns. According to this model, for a black and white halftone, one needs only seven independent representative two-by-two tile 


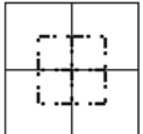

G0

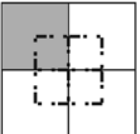

G1

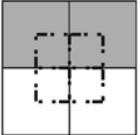

$\mathrm{G} 2$

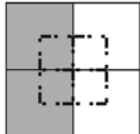

G3

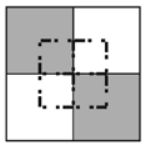

G4

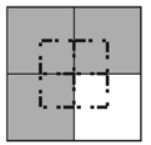

G5

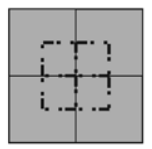

G6

(a)

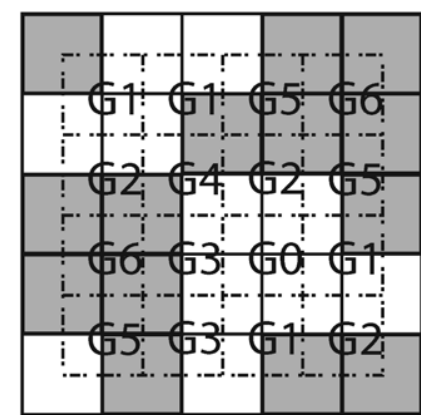

(b)

Figure 3. (a) The seven representative two-by-two pixel tile patterns used for the calibration of the black and white two-by-two prediction model. (b) Example of a halftone with the corresponding mapped representative two-by-two pixel tile patterns.

patterns (Figure 3(a)). Based on these patterns for calibrating the two-by-two dot centering model, one is able to predict all possible configurations of black and white halftones (Fig. 3(b)). These calibration tile patterns together with their symmetrical pairs along the horizontal and vertical axes describe all possible black and white distributions within a two-by-two pixel tile.

The reflectances of the representative two-by-two tile patterns form the two-by-two calibration set. In order to predict the reflectance of a halftone, the corresponding halftone element is analyzed. At each location, the corresponding representative two-by-two tile pattern is found and within the halftone the number of occurrences of each two-bytwo representative tile pattern, including its symmetrical counterparts, is counted. The halftone reflectance $R(\lambda)$ is predicted using a Yule-Nielsen approach:

$$
R(\lambda)=\left(\frac{\sum_{u=0}^{6} i_{u} R_{u}(\lambda)^{1 / n}}{\sum_{u=0}^{6} i_{u}}\right)^{n},
$$

where $i_{u}$ is the number of occurrences of the representative tile pattern $G_{u}$, and $R_{u}$ is its corresponding measured reflection spectrum. The Yule-Nielsen $n$-value accounts for the non-linear relationship between the reflectances of the representative tile patterns and the global reflectance of the analyzed halftone pattern.

\section{Predictive Two-by-Two Dot Centering Model}

The two-by-two dot centering model for color halftones is a straightforward extension of its black and white instance. However, for a given 3- or 4-ink print setup, the two-by-two prediction model requires a large number of measurements to characterize all representative two-by-two tiles. Since there are 4 positions within each two-by-two tile, the number of colorant arrangements within a tile increases exponentially with the number of colorants. In the case of 8 colorants $(c, m, y, b, g, r, k, w)$ there are $8^{4}=4096$ possible colorant arrangements. By accounting for horizontal and vertical symmetries, they are reduced to 1072 independent representative tile patterns. ${ }^{11}$
In order to reduce the number of representative pattern reflectances that need to be measured, we estimate the reflectances of approximately $90 \%$ of the representative two-by-two tile patterns by relying on a small subset $(\approx 10 \%)$ of measured two-by-two tile reflectances. ${ }^{12}$ We assume that within the two-by-two tiles, surface coverages of colorants are equivalent to relative colorant concentrations. We therefore work in the absorptance space and establish a linear relationship between surface coverages $(0,0.25,0.5$, 0.75 and 1) and two-by-two tile absorptances. We convert intrinsic reflectances of the selected subset of two-by-two tile patterns into colorant halftone absorptances:

$$
K(\lambda)=-\frac{1}{2} \ln \left(\frac{\rho_{i}(\lambda)}{\rho_{p}(\lambda)}\right),
$$

where $K(\lambda)$ is the spectral absorptance of the considered two-by-two tile colorant halftone; $\rho_{i}(\lambda)$ and $\rho_{p}(\lambda)$ are the intrinsic reflectances obtained by applying the inverse Saunderson correction ${ }^{13}$ to the measured reflectances $R_{i}(\lambda)$ and $R_{p}(\lambda)$, where subscripts $i$ and $p$ stand respectively for the corresponding two-by-two ink halftone tile and the unprinted paper. The intrinsic reflectance is the reflectance that would be measured from within a surrounding medium having the same index of refraction as the print. The inverse Saunderson correction yielding the intrinsic reflectances $\rho(\lambda)$ as a function of the measured reflectances $R(\lambda)$ is

$$
\rho(\lambda)=\frac{R(\lambda)-K \cdot r_{s}}{1+(1-K) r_{i} \cdot r_{s}+r_{i} \cdot R_{m}(\lambda)-r_{i}-r_{s}},
$$

where $r_{s}$ is the Fresnel specular reflection at the air-print interface, $K$ is the portion of specular reflection captured by the spectrophotometer and $r_{i}$ is the overall Fresnel reflection at the print-air interface. For a print with an assumed refraction index of 1.5 , illuminated by collimated light at $45^{\circ}$, $r_{i}=0.6$ and $r_{s}=0.04$.

By performing a multivariate linear regression between the absorptances and surface coverages of a subset of measured calibration two-by-two tiles, we establish a transformation matrix $\mathbf{M}$ which maps the colorant surface coverages of two-by-two tiles to their spectral absorptance. 


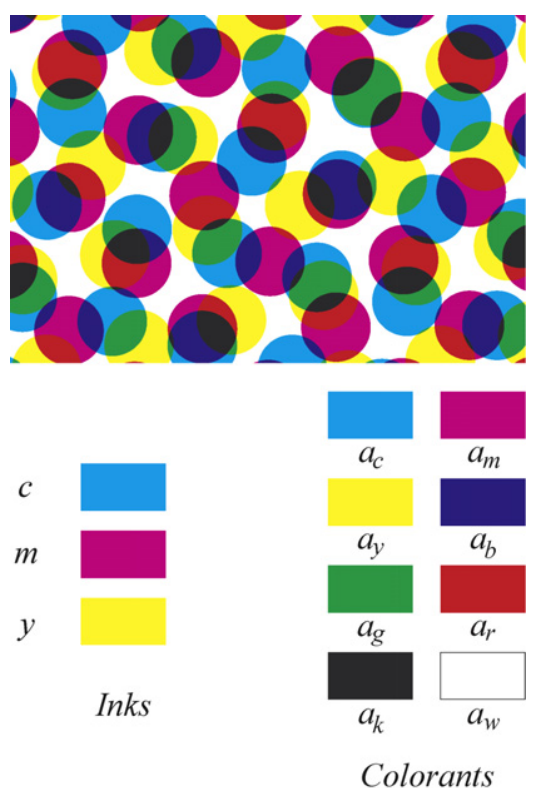

Figure 4. Schematic representation of the Demichel equations. The 8 Neugebaver primaries (Colorants) are formed by paper, solid inks and solid ink superpositions. The colorant surface coverages depend on the surface coverages of the inks.

This matrix is obtained by making $m$ observations using $m$ measured two-by-two tile pattern reflectances $R_{1}(\lambda)$, $R_{2}(\lambda), \ldots, R_{m}(\lambda)$ from which we derive their intrinsic reflectances $\rho_{1}(\lambda), \rho_{2}(\lambda), \ldots, \rho_{m}(\lambda)$ and then their absorptances $K_{1}(\lambda), K_{2}(\lambda), \ldots, K_{m}(\lambda)$. In vector form:

$$
\left[\mathbf{k}_{1}, \mathbf{k}_{\mathbf{2}}, \ldots, \mathbf{k}_{\mathbf{m}}\right]=\mathbf{M}\left[\mathbf{c}_{1}, \mathbf{c}_{2}, \ldots, \mathbf{c}_{\mathbf{m}}\right],
$$

where $\mathbf{k}_{\mathbf{i}}$ is the spectral absorptance vector comprising, e.g., 36 wavelength components created by the $i$ th measured two-by-two calibration tile pattern of surface coverage $\mathbf{c}_{\mathbf{i}}$. In shorter form:

$$
\mathbf{K}=\mathbf{M C},
$$

where the matrix $\mathbf{K}$ is the absorptivity matrix with 36 rows and $m$ columns, i.e., one column per observation, $\mathbf{C}$ is an 8 by $m$ matrix containing for each of the $m$ observations its 8 colorant surface coverages and $\mathbf{M}$ is the 36 row by 8 column transformation matrix. We solve this linear system for the matrix $\mathbf{M}$ by minimizing $\|\mathbf{K}-\mathbf{M C}\|$ according to the least squares approach. Using the transformation matrix $\mathbf{M}$ we predict the unknown absorptances and, consequently, the reflectances of those representative two-by-two patterns that have not been measured. It should be noted that the estimation of the reflectance of representative two-by-two patterns is part of the calibration of the two-by-two prediction model. With the calibration set formed by the measured and predicted representative tile pattern reflectances, the two-by-two dot centering model is used to predict the reflectances of juxtaposed or clustered dot color halftones.

\section{Variants of the Yule-Nielsen Spectral Neugebauer Model}

The spectral Neugebauer model predicts the overall reflectance $R(\lambda)$ of a halftone as a weighted average of the colorant reflectances $R_{i}(\lambda)$. Yule and Nielsen ${ }^{14}$ and Viggiano ${ }^{15}$ proposed to replace this linear relationship with a non-linear power function whose value $n$ is optimized according to a limited number of measured patch reflection spectra:

$$
R(\lambda)=\left(\sum_{i} a_{i} R_{i}(\lambda)^{1 / n}\right)^{n},
$$

where $a_{i}$ is the fractional area coverage of solid colorant (Neugebauer primary) $i, R_{i}(\lambda)$ is its reflectance spectrum and $R(\lambda)$ is the predicted reflectance of the halftone patch. The power functions with values $1 / n$ and $n$ account for the optical dot gain due to lateral propagation of light and to multiple internal reflections between the paper bulk and the print-air interface.

The prediction accuracy of the YNSN model depends on our knowledge of the effective surface coverages $a_{i}$ of the contributing colorants. Effective surface coverages depend on the inks, the paper and their superposition condition. Variants of the YNSN model differentiate themselves according to the approach for calculating the surface coverages of the Neugebauer primaries. We consider three approaches. The first approach called nominal YNSN consists in applying nominal surface coverages. The second approach called independent ink spreading YNSN (IIS-YNSN) considers, for each single ink, a curve mapping nominal to effective surface

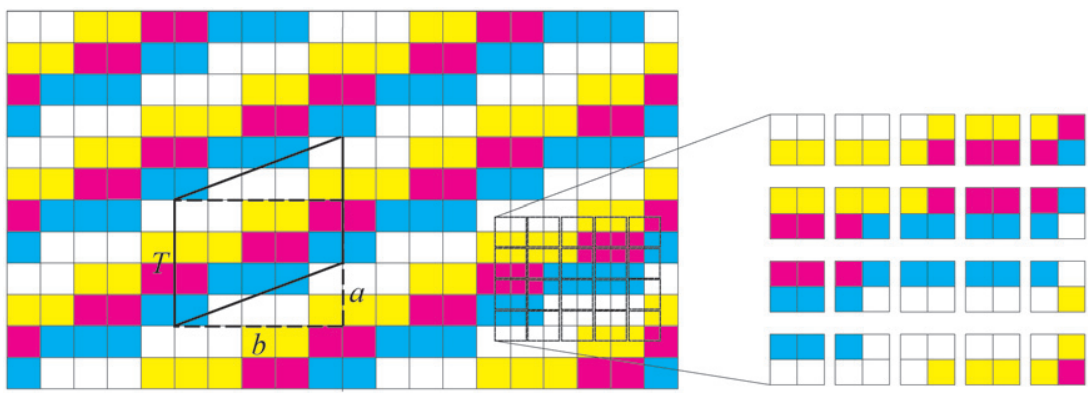

Figure 5. A simple discrete line juxtaposed halftone with three colorants and halftone parameters $a / b=2 / 5$ and $T=4$. The original parallelogram screen element and the equivalent rectangular tile are shown using thick solid and dashed lines, respectively. The two-by-two patterns occurring inside the equivalent rectangular tile are shown by thin solid lines. This tile may be located anywhere within the halftone. 
coverages. These curves are obtained by linear interpolation between fitted surface coverages of halftones printed on paper.

The third approach, called the superposition dependent ink spreading YNSN (SDIS-YNSN) model, relies on ink spreading curves mapping nominal to effective surface coverages for each superposition condition. In order to obtain the effective surface coverages of the inks of a classical three-ink system (cyan, magenta and yellow) as a function of nominal surface coverages (control values), the contributions of the different ink spreading curves are weighted according to the ratio of colorants forming that halftone. $^{4}$

For all three approaches, it is assumed that the cyan, magenta and yellow ink dot layers are laid out independently of each other. The effective surface coverages of the colorants are then obtained from the surface coverages of the inks according to the Demichel equations: ${ }^{3}$

$$
\begin{aligned}
& a_{c}=c(1-m)(1-y), \\
& a_{m}=m(1-c)(1-y), \\
& a_{y}=y(1-c)(1-m), \\
& a_{b}=c m(1-y), \\
& a_{g}=c y(1-m), \\
& a_{r}=m y(1-c), \\
& a_{k}=c m y, \\
& a_{w}=(1-c)(1-m)(1-y),
\end{aligned}
$$

where $c, m$ and $y$ are the surface coverages of the cyan, magenta and yellow partially overlapping ink dots and where $a_{c}, a_{m}, a_{y}, a_{b}, a_{g}, a_{r}, a_{k}$ and $a_{w}$ are the surface coverages of the resulting colorants cyan, magenta, yellow, blue, red, green, black and white, respectively (see Figure 4).

\section{RESULTS AND DISCUSSION Applying the Two-by-Two Model to Discrete Line Juxtaposed Halftones}

To obtain the reflectance of a halftone using the two-by-two dot centering model we need to count the number of occurrences of each representative two-by-two pattern inside a halftone screen element. The screen element generated by our discrete line juxtaposed halftoning is a parallelogram tile whose size depends on two parameters: slope $a / b$ and vertical period $T$, as shown in Figure 5. Any $b \times T$ rectangle forms a tile equivalent to the parallelogram screen element.

\section{Performance of the Two-by-Two Model Compared to YNSN}

In order to assess the performance of the two-by-two spectral prediction model on discrete line juxtaposed halftones, we compare it with the well-known YNSN. To perform a fair comparison, we create juxtaposed halftones with colorant surface coverages meeting the constraints of the YNSN model. While juxtaposed halftoning enables us to directly set the surface coverages of input colorants independently of each other, we chose 8 colorant surface coverages so as to respect the Demichel equations. We convert all combinations of $0,0.25,0.5,0.75$ and 1 of cyan, magenta and yellow inks into surface coverages of the 8 colorants using Eq. (7). This results in 125 test patches. Table $\mathrm{V}$ in appendix B shows an example of colorant surface coverages calculated according to the Demichel equations. These surface coverages are reproduced using discrete line juxtaposed halftoning.

The 125 generated test halftone patches were printed on Canon MP-101 paper. The experiments were carried out on a Canon Pixma Pro9500 inkjet printer at 600 dpi driven in high-quality mode, with a droplet size of 3 producing saturated ink colors. The corresponding solid cyan, magenta and yellow densities were respectively 1.00, 0.80 and 1.08 (DIN 16536-2 density standard). The halftones were generated at two vertical screen periods, $T=7$ pixels yielding a screen frequency of $99 \mathrm{lpi}$, and $T=11$ pixels yielding a screen frequency of $63 \mathrm{lpi}$, for a single discrete line slope $m=4 / 7$. The last column of Table I shows the $n$-values used for the considered models. To obtain the best value, we vary $n$ from 1 to 10 with steps of 0.1 ( $n$-values greater than 10 change the results negligibly).

Table I shows the prediction accuracy for special juxtaposed line halftones obeying the Demichel equations. We considered the variants of the YNSN model as well as the two-by-two dot centering model whose calibration set comprises either completely measured or partly measured and partly predicted patch reflectances.

In order to better appreciate the achievable accuracy of the printing system, Table II shows the prediction accuracy of the same prediction models for a three-ink classical clustered dot rotated screen halftone at 100 lpi. The test set is composed of 125 test patches with the same nominal surface coverages as the ones used for the tests in Table I. For predictions made by the two-by-two dot centering model, we either measure all 1072 representative patterns or measure 100 randomly selected representative patterns and predict the remaining ones.

The results shown in Tables I and II demonstrate that the accuracy of the YNSN model is improved when using single-ink dot gain curves (IIS-YNSN). Applying the SDIS-YNSN model with a separate ink spreading curve per superposition condition does not significantly improve the accuracy, because for this printer the ink spreading curves are similar in the different superposition conditions.

The $n$-value is known to be responsible for the optical dot gain, which occurs when light is propagated from one colorant to other colorants. Since the calibration patterns of the two-by-two model are halftones comprising all possible arrangements of colorants, they automatically account for the optical dot gain with neighboring colorants. Hence, the $n$-value fitted for the two-by-two model according to Eq. (1) is mainly responsible for further non-linear relationships between target halftone reflectance and representative twoby-two pattern reflectances such as variations in ink dot thickness. ${ }^{16}$ In contrast, the SDIS-YNSN $n$-value accounts 
Babaei and Hersch: Spectral prediction of juxtaposed halftones relying on the two-by-two dot centering model

Table I. Prediction accuracy of the two-by-two and YNSN models for discrete line juxtaposed halftoning for 125 test halftones obeying the Demichel equations, on a Canon Pixma Pro9500 at 600 dpi.

\begin{tabular}{llllllll}
\hline Halftone parameters & Prediction model & \# Calib. set & \multicolumn{3}{c}{$\Delta E_{94}$} & \multirow{2}{*}{ n-value } \\
\cline { 3 - 5 } & & & Mean & $95 \%$ & Max & \\
\hline$T=7, m=4 / 7,99$ Ipi & Nominal YNSN & 8 & 2.32 & 6.94 & 12.33 & 10 \\
& IIS-YNSN & 17 & 1.50 & 6.03 & 10.13 & 10 \\
& SDIS-YNSN & 44 & 1.48 & 6.24 & 10.53 & 10 \\
& Predictive 2-by-2 & 100 & 1.39 & 3.32 & 3.99 & 2.4 \\
& Full 2-by-2 & 1072 & 0.70 & 1.68 & 4.27 & 2.5 \\
$T=11, m=4 / 7,63$ Ipi & Nominal YNSN & 8 & 1.84 & 6.26 & 9.52 & 3.9 \\
& IIS-YNSN & 17 & 1.63 & 7.00 & 8.60 & 3.8 \\
& SDIS-YNSN & 44 & 1.51 & 6.51 & 8.47 & 3.9 \\
& Predictive 2-by-2 & 100 & 1.34 & 2.89 & 4.64 & 1.6 \\
& Full 2-by-2 & 1072 & 0.91 & 2.08 & 4.61 & 1.6 \\
\hline
\end{tabular}

Table II. As a reference, prediction accuracy of the two-by-two and YNSN model variants for 125 classical clustered dot halftones, on a Canon PIXMA Pro9500 at 600 dpi.

\begin{tabular}{llllllll}
\hline Halftone parameters & Prediction model & \multirow{2}{*}{ \# Calib. set } & \multicolumn{3}{c}{$\Delta E_{94}$} & \multirow{2}{*}{ n-value } \\
\cline { 3 - 5 } & & & Mean & $95 \%$ & Max & \\
\hline Clustered dot, 100 Ipi & Nominal YNSN & 8 & 1.29 & 2.28 & 2.87 & 10 \\
& IIS-YNSN & 17 & 0.41 & 0.99 & 1.46 & 9 \\
& SDIS-YNSN & 44 & 0.36 & 0.81 & 0.99 & 9.9 \\
& Predictive 2-by-2 & 100 & 1.41 & 3.38 & 3.85 & 2.4 \\
& Full 2-by-2 & 1072 & 0.64 & 1.21 & 1.72 & 2.4 \\
\hline
\end{tabular}

for all phenomena including the optical dot gain. Therefore it is larger than the two-by-two $n$-value.

\section{Test Sets With Freely Chosen Surface Coverages}

Juxtaposed halftones with arbitrary colorant surface coverages do not satisfy the Demichel equations. In this case, for predictions with the YNSN model we assume that the surface coverages of the colorants are equal to the known nominal surface coverages. In Table III we show the prediction accuracy of the nominal YNSN and the two-by-two prediction models for 125 juxtaposed halftones with non-Demichel surface coverages of the 8 Neugebauer primaries. The surface coverages of this test set are obtained by exchanging the old surface coverages of the colorants. We exchanged the surface coverages of cyan and blue as well as of yellow and red, thereby invalidating the Demichel equations (see Table VI in appendix B). As expected, the YNSN model with nominal ink surface coverages does not yield a high prediction accuracy. The two-by-two model performs accurately and offers slightly increased prediction accuracy compared to predictions on patches obeying the Demichel equations. The two-by-two model directly captures the halftone configuration and does not need explicit knowledge about surface coverages and ink spreading.

\section{Test Sets With Custom Inks}

Finally, in order to show the prediction accuracy of a real-life example we test the aforementioned spectral prediction models on the same non-Demichel surface coverages as in the previous section for seven custom inks. In the previous test sets, the blue, green, red and black primaries were obtained by superposition of the original cyan, magenta and yellow inks. In the present test set, in addition to cyan, magenta and yellow we use custom blue, green, red and black inks.

As can be seen from Table IV, the results are similar to those from the previous test sets. The two-by-two model offers more accurate predictions than the nominal YNSN model. However, the predictions obtained by the two-by-two model with all calibration patterns measured (full 2-by-2) are slightly worse that the predictions of the corresponding two-by-two model for three-ink prints. With a larger number of inks, there is more variation in the halftones. The overlaps of neighboring colorants are produced by two different inks and not by two colorants which have in many cases one ink in common.

\section{CONCLUSIONS}

The two-by-two dot centering model does not require explicit knowledge of effective surface coverages and is 
Babaei and Hersch: Spectral prediction of juxtaposed halftones relying on the two-by-two dot centering model

Table III. Prediction accuracy of the two-by-two and YNSN model variants for 125 juxtaposed halftones with non-Demichel surface coverages on a Canon PIXMA Pro9500 at 600 dpi.

\begin{tabular}{|c|c|c|c|c|c|c|}
\hline \multirow[t]{2}{*}{ Halftone parameters } & \multirow[t]{2}{*}{ Prediction model } & \multirow[t]{2}{*}{ \# Calib. set } & \multicolumn{3}{|c|}{$\Delta E_{94}$} & \multirow[t]{2}{*}{$n$-value } \\
\hline & & & Mean & $95 \%$ & $\operatorname{Max}$ & \\
\hline \multirow{3}{*}{$\begin{array}{l}\text { Juxtaposed } T=11, m=4 / 7,63 \mathrm{lpi} \\
\text { with non-Demichel surface coverages }\end{array}$} & Nominal YNSN & 8 & 2.63 & 6.77 & 8.72 & 4.7 \\
\hline & Predictive 2-by-2 & 100 & 1.58 & 3.36 & 4.33 & 1.5 \\
\hline & Full 2-by-2 & 1072 & 0.76 & 1.84 & 2.94 & 1.6 \\
\hline
\end{tabular}

Table IV. Prediction accuracy of the two-by-two and YNSN model variants for 125 juxtaposed halftones with cyan, magenta, blue, green, red and pure black custom inks of the same surface coverages as the ones in Table III on a Canon PIXMA Pro9500 at 600 dpi.

\begin{tabular}{|c|c|c|c|c|c|c|}
\hline \multirow[t]{2}{*}{ Halftone parameters } & \multirow[t]{2}{*}{ Prediction model } & \multirow[t]{2}{*}{ \# Calib. set } & \multicolumn{3}{|c|}{$\Delta E_{94}$} & \multirow[t]{2}{*}{$n$-value } \\
\hline & & & Mean & $95 \%$ & $\operatorname{Max}$ & \\
\hline \multirow{2}{*}{ Juxtaposed $T=11, m=4 / 7,631 \mathrm{pi}$} & Nominal YNSN & 8 & 2.36 & 6.65 & 8.57 & 3.9 \\
\hline & Predictive 2-by-2 & 100 & 1.41 & 3.70 & 7.10 & 1.5 \\
\hline 7 custom inks & Full 2-by-2 & 1072 & 1.24 & 3.20 & 6.91 & 1.6 \\
\hline
\end{tabular}

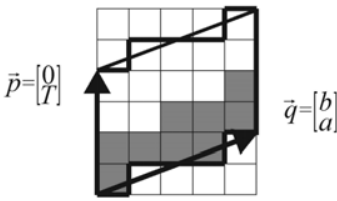

(a)

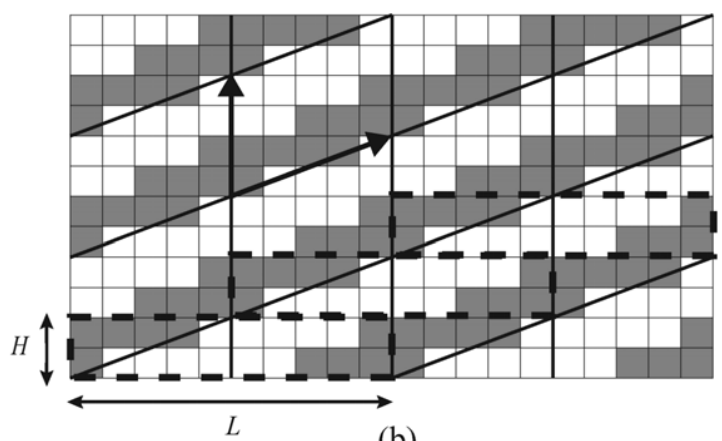

(b)

Figure 6. (a) A parallelogram screen element and its associated vectors and (b) the corresponding screen obtained by replication of the screen element on a $20 \times 12$ output image. The surface coverage is $45 \%$. The vertical thickness $T$ is 4 and the slope is $m=2 / 5$. The equivalent Holladay tile $(H, L)$ and its replications are also shown.

therefore a reliable model to predict the reflection spectra and colors of juxtaposed halftones. Yule-Nielsen modified spectral Neugebauer models cannot be used for predicting the color of juxtaposed halftones. The reason is that the effective surface coverages of the juxtaposed and slightly superposed contributing colorants are unknown. Small overlaps of neighboring colorants due to misregistration as well as variations in colorant ink thicknesses make it difficult to estimate their effective surface coverages. Even on juxtaposed test samples specially conceived to satisfy the Demichel equations, the two-by-two dot centering model offers higher prediction accuracies than the YNSN model variants.

\section{ACKNOWLEDGMENT}

The authors would like to thank Dr. Romain Rossier for the color prediction software. Thanks are due to the anonymous reviewers for their very useful comments. The authors are also grateful for the Swiss National Science Foundation grant no. 200021-143501/1.

\section{Appendix A. Discrete Line Juxtaposed Halftoning}

Let us explain how to generate discrete screen elements made of discrete lines of subpixel thickness and how to halftone an input grayscale image. The screen element is a discrete parallelogram whose surface is segmented into black and white parts according to the desired rational ratio of black and white surface coverages. Parallelogram screen elements are created using discrete lines of appropriate thicknesses, at subpixel precision.

A set $D$ of points $(x, y)$ in $\mathbb{Z}^{2}$ belongs to the discrete line if and only if each member of this set satisfies

$$
\gamma \leq a x-b y<\gamma+w
$$

where $a$ and $b$ are integer values and define the line's rational slope, $\gamma \in \mathbb{Z}$ indicates its position in the plane and $w \in \mathbb{Z}$ determines its thickness. Due to symmetry it is enough to consider the case where $|a|<|b|$, i.e. the line's absolute slope is smaller than 1 . The arithmetic thickness parameter $w$ controls the vertical thickness and the connectivity of the line. If $w<|b|$, the line is a disconnected thin line; if $w=|b|$, 
Babaei and Hersch: Spectral prediction of juxtaposed halftones relying on the two-by-two dot centering model

Table V. Demichel surface coverages of the 8 Neugebauer primaries for a subset of ink surface coverages.

\begin{tabular}{|c|c|c|c|c|c|c|c|c|c|c|}
\hline \multicolumn{3}{|c|}{ Inks } & \multicolumn{8}{|c|}{ Colorants } \\
\hline$c$ & $m$ & $y$ & $a_{c}$ & $a_{m}$ & $a_{y}$ & $a_{b}$ & $a_{g}$ & $a_{r}$ & $a_{k}$ & $a_{W}$ \\
\hline 0 & 0 & 0 & 0 & 0 & 0 & 0 & 0 & 0 & 0 & 1 \\
\hline $1 / 4$ & $3 / 4$ & $3 / 4$ & $1 / 64$ & $9 / 64$ & $9 / 64$ & $3 / 64$ & $3 / 64$ & $27 / 64$ & $9 / 64$ & $3 / 64$ \\
\hline $1 / 2$ & $1 / 2$ & $1 / 2$ & $1 / 8$ & $1 / 8$ & $1 / 8$ & $1 / 8$ & $1 / 8$ & $1 / 8$ & $1 / 8$ & $1 / 8$ \\
\hline $3 / 4$ & $1 / 4$ & $2 / 4$ & $18 / 64$ & $2 / 64$ & $6 / 64$ & $6 / 64$ & $18 / 64$ & $2 / 64$ & $6 / 64$ & $6 / 64$ \\
\hline 1 & 1 & 1 & 0 & 0 & 0 & 0 & 0 & 0 & 1 & 0 \\
\hline
\end{tabular}

Table VI. Non-Demichel surface coverages of the 8 Neugebauer primaries obtained by exchanging the surface coverages of cyan and blue and of yellow and red.

\begin{tabular}{llllllll}
\hline \multicolumn{7}{c}{ Colorants } \\
\hline$a_{c}$ & $a_{m}$ & $a_{y}$ & $a_{b}$ & $a_{g}$ & $a_{r}$ & $a_{k}$ & $a_{W}$ \\
\hline 0 & 0 & 0 & 0 & 0 & 0 & 0 & 1 \\
$3 / 64$ & $9 / 64$ & $27 / 64$ & $1 / 64$ & $3 / 64$ & $9 / 64$ & $9 / 64$ & $3 / 64$ \\
$1 / 8$ & $1 / 8$ & $1 / 8$ & $1 / 8$ & $1 / 8$ & $1 / 8$ & $1 / 8$ & $1 / 8$ \\
$6 / 64$ & $2 / 64$ & $2 / 64$ & $18 / 64$ & $18 / 64$ & $6 / 64$ & $6 / 64$ & $6 / 64$ \\
0 & 0 & 0 & 0 & 0 & 0 & 1 & 0 \\
\hline
\end{tabular}

the line is called a naive digital line and has exactly the vertical thickness of 1 ; if $w>|b|$, the line has a vertical thickness greater than 1 .

The sides of the parallelogram forming the screen element are defined by vectors $\mathbf{p}=[0 T]^{T}$ and $\mathbf{q}=[b a]^{T}$ (Figure 6(a)). The parameter $T$ defines the vertical thickness of the discrete line. Hence, within the parallelogram screen, a discrete line segment forming the surface of a colorant may have a vertical thickness between 0 and $T$ in steps of $1 / b$. As an example, Fig. 6(b) shows a parallelogram screen with $45 \%$ surface coverage.

In order to establish the monochrome screen element library, the bilevel screen elements are generated level by level by creating each time within the parallelogram tile a "black" discrete line having a vertical thickness between 0 and $T$, with $b \cdot T$ different possible thicknesses. The discrete line thicknesses are 0 for a surface coverage of $0,1 / b$ for a surface coverage of $(1 / b) \cdot T, 2 / b$ for a surface coverage of $(2 / b) \cdot T, \ldots, 1$ for a surface coverage of $1 / T$ and $T$ for a surface coverage of 1 .

Once the screen element library is created, halftoning is performed by traversing the output halftone image scanline by scanline and pixel by pixel. The image plane is paved by replicating the parallelogram screen element along its side vectors $[b a]^{T}$ and $[0 T]^{T}$. Instead of using parallelogram screen elements, we can produce equivalent rectangular screen elements tiling the plane according to Holladay's algorithm. ${ }^{17}$ Given a discrete parallelogram with sides given by vectors $\mathbf{p}$ and $\mathbf{q}$, Holladay's algorithm yields an equivalent $L$ by $H$ rectangular tile (Fig. 6(b)). Paving the image plane with this rectangular tile is equivalent to paving the plane with the original discrete parallelogram. At halftoning time, for each output pixel position we find the corresponding location in the input continuous tone image.
The gray value at that location determines an entry within the screen element library. The current output pixel location determines the location within the screen element whose element (black or white) is to be copied into the current output pixel.

The two-by-two spectral prediction model offers accurate predictions on juxtaposed inkjet halftones both for classical cyan, magenta and yellow inks and for custom inks. In the future, we intend to verify the prediction accuracy of this model for juxtaposed halftoning of special inks such as metallic inks and luminescent inks.

\section{Appendix B. Examples of Surface Coverages of Inks and Colorants}

Table $\mathrm{V}$ gives the surface coverages of the colorants (Neugebauer primaries) for given surface coverages of inks according to the Demichel equations (7). Table VI shows the corresponding non-Demichel surface coverages of Neugebauer primaries obtained by exchanging the surface coverages of the cyan and blue and of the yellow and red colorants.

\section{REFERENCES}

${ }^{1}$ R. Bala, "Device characterization," Digital Color Imaging Handbook, edited by G. Sharma (CRC Press, 2003), pp. 269-382.

${ }^{2}$ R. Balasubramanian, "Optimization of the spectral Neugebauer model for printer characterization," J. Electron. Imaging 8, 156-166 (1999).

${ }^{3}$ D. R. Wyble and R. S. Berns, "A critical review of spectral models, applied to binary color printing," Color Res. Appl. 25, 4-19 (2000). With the Demichel equations originally published in M. E. Demichel, Procédé, Vol. 26, pp. 17-21, 1924.

${ }^{4}$ R. D. Hersch and F. Crété, "Improving the Yule-Nielsen modified spectral Neugebauer model by dot surface coverages depending on the ink superposition conditions," Proc. SPIE 5667, 434-445 (2005).

${ }^{5}$ V. Ostromoukhov and R. D. Hersch, "Multi-color and artistic dithering," Proc. SIGGRAPH 99, Computer Graphics Proceedings, Annual Conf. Series (1999), pp. 425-432.

${ }^{6}$ R. D. Hersch, P. Donzé, and S. Chosson, "Color images visible under UV light," ACM. Trans. Graphics (Proc. ACM SIGGRAPH) 22, 427-436 (2007).

${ }^{7}$ J. Morovic, P. Morovic, and J. Arnabat, "HANS: Controlling ink-jet print attributes via Neugebauer primary area coverages," IEEE Trans. Image Process. 21, 688-696 (2012).

${ }^{8} \mathrm{~V}$. Babaei and R. D. Hersch, "Juxtaposed color halftoning relying on discrete lines," IEEE Trans. Image Process. 22, 679-686 (2013).

${ }^{9} \mathrm{~J}$. P. Reveillès, "Géométrie discrète, calcul en nombres entiers et algorithmique," Ph.D. dissertation (University of Louis Pasteur, Strasbourg, 1991).

${ }^{10} \mathrm{~S}$. G. Wang, "Two-by-two centering printer model with Yule-Nielsen equation," Proc. IS\&T's NIP14: Int'l. Conf. on Digital Printing Technol. (IS\&T, Springfield, VA, 1998), pp. 302-305. 
${ }^{11}$ C. Hains, S. G. Wang, and K. Knox, "Digital color halftones," Digital Color Imaging Handbook, edited by G. Sharma (CRC Press, 2003), pp. 470-479.

${ }^{12}$ V. Babaei, R. Rossier, and R. D. Hersch, "Reducing the number of calibration patterns for the two-by-two dot centering model," Proc. SPIE 8292, 1-9 (2012).

${ }^{13}$ J. L. Saunderson, "Calculation of the color of pigmented plastics," J. Opt. Soc. Am. A 32, 727-736 (1942).
${ }^{14}$ J. A. C. Yule and W. J. Nielsen, "The penetration of light into paper and its effect on halftone reproductions," Proc. TAGA 3, 65-76 (1951).

${ }^{15}$ J. A. S. Viggiano, "Modeling the color of multi-colored halftones," Proc. TAGA 44-62 (1990).

${ }^{16} \mathrm{M}$. Hébert and R. D. Hersch, "Analyzing halftone dot blurring by extended spectral prediction models," J. Opt. Soc. Am. A 27, 6-12 (2010).

${ }^{17}$ T. M. Holladay, "An optimum algorithm for halftone generation for displays and hard copies," Proc. Soc. Infor. Disp. 21, 185-192 (1980). 